\title{
LA RÉCEPTION DE PAULO FREIRE FACE AU NÉOCONSERVATISME EN FRANCE
}

\author{
A RECEPÇÃO DE PAULO FREIRE DIANTE DO NEOCONSERVADORISMO NA \\ FRANCA
}

PAULO FREIRE'S RECEPTION BEFORE NEOCONSERVATORISM IN FRANCE

\author{
Irène Pereira \\ Université Paris-Est, LIS (EA 4395) UPEC, F-94010, Créteil, France \\ irene.pereira@u-pec.fr
}

\begin{abstract}
Résumé: TrèSconnu en France dans les années 70 du XXe siècle, Paulo Freire était pratiquement oublié, à l'exception de vagues références et slogans dans l'éducation populaire. La reprise de sa pensée sur le sol français a commencé en 2015. Ce sauvetage était dû à la les difficultés rencontrées par les militants et les penseurs de gauche face au néolibéralisme et au néoconservatisme et auxquelles l'héritage freirien offre des réponses appropriées et opportunes. Ainsi, les défenseurs des politiques d'émancipation trouvent soutien et se sentent mieux instrumentalisés par les idées de Paulo Freire pour faire face, avec efficience et efficacité révolutionnaire, aux menaces «bancaires»du projet politico-pédagogique néolibéral et conservateur.
\end{abstract}

Mots-clés: Émancipation. Néolibéralisme. Néoconservatisme. Paulo Freire.

Resumo: Muito conhecido na França nos anos 70 do século XX, Paulo Freire ficou praticamente esquecido, a não ser por referências vagas e slogans na educação popular, tendo se iniciado a recuperação de seu pensamento em solo francês a partir de 2015. Este resgate se deveu às dificuldades enfrentadas pelos militantes e pelos pensadores de esquerda diante do neoliberalismo e do neoconservadorismo e às quais o legado freiriano oferece respostas adequadas e oportunas. Assim os defensores de políticas emancipadoras encontram apoio e se sentem melhor instrumentalizados pelas ideias de Paulo Freire para enfrentar, com eficiência e eficácia revolucionária, às ameaças "bancárias" do projeto político-pedagógico neoliberal e conservador.

Palavras Chave: Emancipação. Neoliberalismo. Neoconservadorismo. Paulo Freire.

Abstract: Very well known in France in the 70's of the 20th century, Paulo Freire was practically forgotten, except for vague references and slogans in popular education, having started to recover his thinking on French soil since 2015. This rescue was due to the difficulties faced by militants and leftwing thinkers in the face of neoliberalism and neoconservatism and to which the Freirian legacy offers appropriate and timely responses. Thus, defenders of emancipatory policies find support and feel better instrumentalized by Paulo Freire's ideas to face, with efficiency and revolutionary effectiveness, the "banking" threats of the neoliberal and conservative political-pedagogical project.

Keywords: Emancipation. Neoliberalism. Neoconservatism. Paulo Freire.

Citer - (ABNT NBR 6023:2018)

PEREIRA, Irène. La réception de Paulo Freire face au néoconservatisme en France. Eccos - Revista Cientifica, São Paulo, n. 52, p. 1-14, e17098, janv./mars. 2020. Disponível em:

https://doi.org/10.5585/eccos.n52.17098. 
En France, Paulo Freire a bénéficié d'une importante surtout réception durant les années 1970. Sans doute était-ce lié entre autres à sa proximité géographique au moment où il était en exil à Genève. Avant 2015, date où nous avons commencé plus particulièrement à travailler sur son œuvre, il n'était plus qu'une référence vague surtout utilisée dans les milieux de l'éducation populaire, résumé à quelques citations réduites à des slogans. Il n'existait que très peu de travaux universitaires qui lui étaient encore consacrés et ceux-ci n'avaient pas de réception auprès du grand public.

Nous avons depuis essayé quelque peu de combler cette lacune. D'une part, avec des productions accessibles au grand public, qui ont bénéficié d'une assez large réception comme: Paulo Freire, Pédagogue des opprimé-e-s (PEREIRA, 2017); Pédagogies critiques (DE COCK ; PEREIRA, 2019). D'autre part, avec des ouvrages de stature plus universitaire destinés à un public académique: Philosophie critique en éducation (PEREIRA, 2018) et Anthologie internationale de Pédagogie critique (PEREIRA, 2019).

La question qui se pose alors, c'est pourquoi avoir décidé de s'intéresser à l'œuvre relativement oubliée en France de Paulo Freire. Il nous est apparu que la pédagogie critique de Paulo Freire nous permettait de sortir d'un certain nombre de difficultés théoriques et pratiques dans le cadre d'un contexte à la fois néolibéral et néoconservateur.

Au cours de ce texte, nous souhaitons souligner, à travers parfois quelques parallèles avec la situation actuelle au Brésil, les thématiques qui nous ont semblé justifier de porter un intérêt tout à fait spécifique à l'œuvre de Paulo Freire: Comment distinguer les pédagogies néolibérales des pédagogies émancipatrices? Comment résister à la domination de la raison instrumentale en pédagogie? Comment résister à la montée des thématiques néoconservatrices dans le champ de l'éducation? C'est donc autour de ces trois axes que nous allons organiser notre texte.

\section{L’Émancipation Sociale Pédagogique face au Néolibéralisme}

\subsection{Néolibéralisme et Néoconservatisme}

Certains politologues ont parfois cru qu'il y avait un lien intrinsèque entre économie de marché et démocratie libérale. Néanmoins, les exemples ne manquent pas pour contredire une telle thèse: le Chili de Pinochet, Singapour ou d'autres micro-Etats d'Asie du Sud-Est... L'arrivée au pouvoir de Jair Bolsonaro montre une nouvelle fois la compatibilité entre droite 
autoritaire et néolibéralisme. Dans son programme électoral, Bolsonaro ne cesse de louer les bienfaits du néolibéralisme. Sur le plan éducatif, il met justement en avant les exemples de ces États autoritaires d'Asie du Sud-Est qui sont en tête des classements PISA. Le système éducatif doit être entièrement orienté vers la formation de futurs producteurs adaptés aux demandes du marché néolibéral.

En France, la thématique néolibérale est fortement présente dans le discours du Président de la République Emmanuel Macron. Il s'agit de faire de la France une « start-up nation ». Ainsi a-t-il déclaré en anglais, par exemple, le 15 juin 2017 sur son compte twitter : «I want France to be a start-up nation. A nation that thinks and moves like a start-up. » Il est fort à supposer que Jean-Michel Blanquer, le Ministre de l'Education nationale, tienne compte de cette orientation. Néanmoins pour comprendre les difficultés que pose cette visée, il est nécessaire de comprendre comment était structuré le débat pédagogique en France depuis au moins les années 1990.

\subsection{Le Débat entre Traditionaliste et Moderniste}

Le débat s'est structuré principalement autour de l'opposition entre républicains et pédagogues qui a conduit à figer les positions, mais surtout à introduire un certain nombre d'ambiguïtés.

D’un côté, se situent ceux que Grégory Chambat a appelé les «réacpublicains » (CHAMBAT, 2016): jeu de mots entre « réactionnaires » et « républicains » opposés aux pédagogues favorables aux pédagogies nouvelles. Ces réac-publicains sont présentés par l'auteur comme étant favorables à la fois à une pédagogie traditionnelle et à une vision autoritaire et néolibérale de l'école. La thèse de Grégory Chambat, c'est qu'il y aurait un lien intrinsèque entre trois éléments: l'autoritarisme, le néolibéralisme et les pédagogies traditionnelles. Cette thèse a l'avantage de présenter un cadre simple et rassurant pour la pensée de gauche.

Mais la réalité est sans doute plus complexe. En effet, il est apparu que des franges de la gauche se sont mises à porter des critiques contre les pédagogies nouvelles. Ces critiques de gauche sont de deux ordres. La première consiste à mettre en avant les connivences entre les pédagogues et le néolibéralisme. Ainsi, on a vu en France des pédagogues défendre au côté des néolibéraux l'introduction par exemple de la notion de compétence portée par l'OCDE. Cette orientation a par exemple été critiquée par Christian Laval (LAVAL, 2003), ou encore Nico Hirtt (HIRTT, 2009). Un deuxième type de critique que l'on trouve à gauche face aux 
pédagogies nouvelles, c'est que celles-ci seraient en réalité plus favorables aux enfants des classes moyennes supérieures et contribueraient davantage que les pédagogies explicites à creuser les inégalités sociales. Ces sociologues des inégalités sociales reprennent bien souvent l’opposition développée par Basile Bernstein entre «pédagogies visibles » et «pédagogies invisibles ».

Le problème qui est posé est donc le suivant : le capitalisme est-il intrinsèquement lié à une pédagogie traditionnelle ou peut-il s'accorder avec des pédagogies nouvelles?

Notre thèse, c'est que la réalité du capitalisme industriel et néolibéral est ambiguë. Ainsi, le MEDEF (syndicat des patrons) dans une contribution de 2012 sur l'école (MEDEF, 2012) semble dégager deux orientations. La première consiste dans la formation de travailleurs peu qualifiés dont il est nécessaire qu'ils maîtrisent les «savoirs fondamentaux »: lire, écrire, compter. Pour cela, une pédagogie d'inspiration behavioriste pourrait être tout à fait suffisante. Mais en même temps, il apparaît que pour la formation des cadres d'autres compétences sont nécessaire : la créativité, la coopération, la communication... Ce que l'on appelle les soft-skills. Pour cela des pédagogies nouvelles pourraient être bien plus adaptées.

Néanmoins, les tenants d'une vision binaire du champ pédagogique ont dû se résoudre à modifier leurs analyse en constatant qu'effectivement le gouvernement actuellement en place faisait un éloge appuyé de la pédagogie Montessori. En effet, le Ministre de l'Éducation nationale ne cesse de vanter «l'esprit de Montessori » qu'il veut introduire dans les écoles publiques françaises. Face à cela, Philippe Meirieu, important pédagogue français, a répondu dans un ouvrage qui s'intitule La Riposte (MEIRIEU, 2018). Il y distingue entre trois courants: les anti-pédagogues, les pédagogues et les hyper-pédagogues. Le Ministre actuel ne serait donc pas anti-pédagogue, un réac-publicain, mais plutôt un hyper-pédagogue. De son côté, Grégory Chambat (CHAMBAT ; CHEVARIN, 2019) a aussi fait évoluer sa position en considérant qu'il existe un courant mêlant pédagogies alternatives et école privée qui s'attaque à l'école publique. Face à cela, la pédagogie de Célestin Freinet constituerait un rempart: une pédagogie alternative publique.

\subsection{Pédagogies Conservatrice, Pédagogies Libérales et Pédagogies Radicales.}

Ce qui nous a semblé intéressant avec Paulo Freire, et des auteurs qui se réclament de lui, comme Henry Giroux, a été leur positionnement très hostile au néolibéralisme. On ne trouve pas chez Paulo Freire de positions ambivalentes et de collusion avec le néolibéralisme. On trouve chez Henri Giroux (GIROUX, 2018) une distinction qui nous a semblé tout à fait 
éclairante entre pédagogies conservatrices, pédagogies libérales et pédagogies radicales. Il considère que les deux premières se distinguent de la troisième par le fait que la pédagogie radicale prend en compte la dimension politique de la pédagogie: cela consiste à considérer que la pédagogie doit étudier et lutter explicitement contre les rapports sociaux de pouvoir.

Lorsqu'on lit l'ouvrage de Philippe Meirieu, La Riposte, la finalité assignée à la pédagogie reste assez générale et en réalité suffisamment consensuel pour qu'un hyperpédagogue néolibéral puisse s'y retrouver: «former des sujets capables de résister à la toutepuissance pulsionnelle, d'oser penser par eux-mêmes, et de s'engager ensemble dans la construction démocratique du bien commun »(MEIRIEUX, 2018, p. 186). Citation qu'il reprend en réalité du philosophe Alain qui n'était pas particulièrement connu pour ses positions orientées vers l'émancipation sociale.

Au contraire, Paulo Freire, dans Pédagogie de l'autonomie, soutient clairement la lutte contre les discriminations, les inégalités sociales et le capitalisme: «Je suis professeur pour soutenir constamment la lutte contre toute forme de discrimination, contre la domination économique des individus ou des classes sociales. Je suis professeur pour me manifester contre l'ordre capitaliste vigoureux qui inventa cette aberration totale: la misère dans l'opulence » (FREIRE, 2006, p. 116).

La thèse que nous défendons, c'est que nous avons besoin, en France, de la radicalité des thèses de Paulo Freire pour éviter toute collusion des pédagogies émancipatrices avec le néolibéralisme et distinguer plus clairement entre pédagogies néolibérales et pédagogies d'émancipation sociale.

\section{La Lutte contre la Domination de la Rationalité Technique en Pédagogie}

\subsection{L'Accentuation de la Rationalité Technique en Pédagogie}

Comme l'ont montré certains philosophes contemporains, comme Eric Sadin, le néolibéralisme est intrinsèquement lié au développement d'un technolibéralisme s'appuyant sur les nouvelles technologies numériques. Ce phénomène accentue une tendance déjà présente dans la modernité et décrite par l'Ecole de Francfort qui est la domination de la raison instrumentale. 
L'accentuation de la domination de la rationalité instrumentale en pédagogie actuellement en France prend plusieurs formes et reflète en particulier la mise en œuvre d'un positivisme issu de l'Evidence Based Education.

Cela se traduit actuellement par plusieurs orientations. En janvier 2019, le Ministre de l'Education nationale, Jean-Michel Blanquer nomme un Conseil Scientifique de l'Education. En son sein, on note une forte représentation des sciences cognitives et en particulier des neurosciences. C'est en réalité, un courant bien précis des neurosciences, contesté par d'autres comme Olivier Houdé ou Catherine Vidal, celui incarné par Stanislas Dehaene qui est mis en avant.

Au Printemps 2018, le Ministre diffuse une série de recommandations très précises sur l'enseignement de la lecture dans les classes de Cours Préparatoire en s'appuyant en particulier sur les neurosciences. Le déchiffrement y prend une place considérable dans la définition de l'apprentissage de la lecture au détriment d'une réflexion sur la compréhension.

A la rentrée 2018, et au cours de l'hiver 2019, les élèves de CP et CE1 sont soumis à des tests standardisés qui doivent évaluer leur niveau dans les apprentissages fondamentaux.

Enfin, pour citer un dernier exemple, le Ministre supprime le CNESCO, organisme indépendant de l'évaluation du système scolaire, pour prévoir la création d'un autre organisme d'évaluation qui dépendra plus directement du Ministère de l'Education nationale.

\subsection{La Critique de la Domination de la Raison Instrumentale en Pédagogie}

En septembre 2018, nous avons publié un livre, s'appuyant sur Paulo Freire, et intitulé Bréviaire des enseignant-e-s - Science, éthique et pratique professionnelle - (PEREIRA, 2018) pour critiquer cette domination de la raison instrumentale en éducation.

Paulo Freire a effectué dans plusieurs de ses écrits une critique de la domination de la raison technicienne en pédagogie. On peut en particulier citer des passages de Pédagogie de l'indignation:

\footnotetext{
C'est dans ce sens, entre autres, que la pédagogie radicale n'a jamais pu faire aucune concession aux astuces du «pragmatisme » néolibéral qui réduit la pratique éducative à un entraînement techno-scientifique des apprenants. A un entraînement et non à une formation. La nécessaire formation technico-scientifique des apprenants quand elle se base sur la pédagogie critique n'a rien à voir avec une vision étroitement techniciste et scientifique qui caractérise le simple entraînement. C'est pour cela que l'éducatrice progressiste, capable et sérieuse, non seulement doit bien enseigner sa discipline, mais inciter l'apprenant à penser de manière critique la réalité sociale, politique et historique (FREIRE, 2000, p. 13).
} 
La question de la domination de la raison instrumentale apparaît déjà dans l'importance données aux moyens au détriment des finalités. C'est un point souligné également par Adorno :

Il y a d'autre part dans la relation actuelle à la technique, quelque chose d'exagéré, d'irrationnel, de pathogène. C'est le "voile technologique". Les hommes ont tendance à prendre la technique pour la chose elle-même, comme une fin en soi, possédant sa force propre, et ils oublient ainsi qu'elle est un prolongement de l'homme. Les moyens - et la technique est l'ensemble des moyens visant la conservation de l'espère humaine - sont fétichisés, parce que les fins, une vie digne de l'homme, sont cachées et séparées de la conscience de l'homme (ADORNO, 1984, p. 246-247).

Il est tout à fait significatif que le Ministre actuel puisse prendre ses sources dans des méthodes d'inspiration éloignées: des approches technoscientifiques incluant un entraînement behavioriste, ou également des techniques Montessori. Cela s'explique par le fait que ce ne sont pas directement les techniques qui induisent une finalité idéologique. Les méthodes sont des moyens utilisés en vue d'une fin. La scientificité des méthodes sert ici, comme le souligne Adorno, à masquer les finalités. Il s'agit dans ce cas de la formation à des compétences qui favorisent l'employabilité. Cette domination de la raison instrumentale conduit à ne pas poser publiquement le débat démocratique des finalités de l'institution scolaire et de la vision éducative qu'elle doit porter.

En soi, les techniques pédagogiques ne permettent pas à elles seules indépendamment des finalités qui les orientent de déterminer ce qu'est une pédagogie émancipatrice. De ce fait, par exemple les pédagogies coopératives, qui prétendent en France s'inspirer de Freinet, peuvent être promues par des personnes qui ne refusent pas de montrer en quoi ce type de pédagogies peuvent être en adéquation avec les finalités néolibérales (TOLINI, 2018).

Mais la critique qu'implique la domination de la raison instrumentale en pédagogie ne concerne pas uniquement la question de l'occultation de la réflexion sur les finalités au détriment des moyens, mais elle passe également par une critique d'une conception uniquement instrumentale des moyens. C'est ce qu'implique la distinction entre poièsis et praxis. Ainsi, Paulo Freire a mis en avant dans Pédagogie des opprimés que la pédagogie était une praxis (action-réflexion). La notion de poièsis implique une réflexion sur l'efficacité des moyens pour atteindre une fin. La pédagogie relève alors de la technique. Par exemple, chez Célestin Freinet, le matérialisme pédagogique met l'accent sur les techniques. Et de manière générale, il est à constater une dérive technicienne y compris dans des pédagogies qui ont mis en avant une finalité émancipatrice. Cette mise en avant de la technicité de la pédagogie a été considérée comme un moyen de s'opposer à une pédagogie verbaliste qui ne se distinguait pas de la philosophie de l'éducation. Dans cette pédagogie verbaliste, les universitaires affirmaient de 
grands principes et de grandes finalités dans leurs cours de pédagogie, mais sans donner les moyens de les mettre en œuvre.

Dans Pédagogie de l'autonomie, Paulo Freire insiste sur la dimension éthique de l'action enseignante. Ce que certains éthiciens appellent «l'agir éthique » (St-Vincent, 2015). En cela, il rompt avec une vision techniciste de la pédagogie. L'action pédagogique ne consiste pas à mettre en œuvre des moyens efficaces pour atteindre une fin, fut-elle émancipatrice. En effet, il ne peut pas y avoir d'action pédagogique émancipatrice sans une continuité entre la fin et les moyens. Cela signifie, que si l'éducation vise l'humanisation, la pédagogie ne peut pas mettre en œuvre des moyens qui sont déshumanisants. L'élève ne peut pas être traité comme une machine à apprendre, mais il doit être considéré comme un sujet. L'agir pédagogique ne relève pas de l'agir stratégique ou instrumental, mais d'un agir éthique.

Une pédagogie émancipatrice est donc une pédagogie qui lutte contre la déshumanisation de l'humain tant dans ses finalités que dans ses moyens. Dans ses finalités, il s'agit de s'opposer au capitalisme, au patriarcat ou encore à la colonialité du pouvoir qui conduisent à réifier l'être humain afin de pouvoir l'exploiter, le réduire à un objet de profit. Dans ses moyens, il s'agit d'une pédagogie qui ne considère pas que tous les moyens sont bons pour atteindre une finalité. Mais que les moyens pédagogiques doivent être en accord avec la finalité, à savoir l'humanisation.

\section{L'École Publique à l'Épreuve du Néoconservatisme}

\subsection{L'École Publique Face aux Croisades contre le Genre}

En janvier 2014, un programme scolaire visant à lutter contre les stéréotypes de genre, intitulé l'ABCD de l'égalité, a été l'objet d'une campagne de diffamation dans les réseaux sociaux. Les opposants, issus en particulier de mouvements religieux, ont fait courir le bruit auprès des parents que l'école voulait transformer leurs enfants en homosexuels ou en transgenre. Les détracteurs expliquaient également que l'on allait se livrer à des enseignements à caractère sexuels dès la maternelle: apprentissage de la masturbation, visionnement d'images à caractère pornographiques, usages de sextoys... Cette campagne a abouti au fait que des parents effrayés ont retiré leurs enfants de l'école.

Pour mettre fin à la polémique, le gouvernement a suspendu l'ABCD de l'égalité et depuis évite l'utilisation du terme «genre» dans les textes scolaires. En effet, en France, comme 
ailleurs en Europe ou en Amérique Latine, les groupes religieux fondamentalistes se livrent à une croisade contre la «théorie du genre ».

Comme nous l'avons souligné, un des points qui nous paraît intéressant chez Paulo Freire, c'est qu'il assigne entre autres à l'école de devoir lutter contre « les discriminations de genre ». Cela nous a conduit à nous intéresser à la réception dans différents pays de pédagogies se situant dans la continuité de Paulo Freire qui abordent ces problématiques: «la pédagogie féministe » (SOLAR, 2019 ; COLLET, 2019), « la pédagogie queer », « la pédagogie critique de la norme » (DEVIEILHE, 2019).

Il nous apparaît en effet comme une des missions d'une pédagogie d'émancipation sociale de développer au sein de l'institution scolaire publique ces types de pédagogies qui actuellement sont peu développées en France.

\subsection{L'Éducation Nationale et la Question du Racisme Systèmique}

En décembre 2017, le syndicat d'enseignants, dont je suis membre, Sud Education 93, décide d'organiser un stage pour s'interroger sur l'existence d'un racisme institutionnel au sein de l'Éducation Nationale. Le terme qui est employé dans la brochure du stage est « Racisme d'État ». Le stage comprend également certains ateliers en non-mixité de personnes «racisées » (ce qui veut dire qui subissant le racisme). Ces ateliers, comme dans le cas des groupes de conscience féministes, ont pour objectif de permettre aux personnes s'estimant victimes de racisme institutionnel de pouvoir échanger sur leur vécu.

Un mois avant le stage une polémique éclate sur les réseaux sociaux. Le Ministre de l'Education Nationale, Jean-Michel Blanquer, déclare le stage «inconstitutionnel » et porte plainte contre l'organisation syndicale pour diffamation pour avoir utilisé l'expression «racisme d'Etat».

Il faut savoir que si la notion de «Racisme d'État » ou même de racisme institutionnel n'est pas reconnue en France dans le droit, elle figure sous le terme de racisme systèmique dans la législation de la Province de l'Ontario au Canada:

Le racisme systémique se manifeste lorsqu'une institution ou un ensemble d'institutions agissant conjointement crée ou maintient une iniquité raciale. Cette attitude n'est pas toujours intentionnelle et ne signifie pas nécessairement que le personnel de l'organisme concerné est raciste. C'est souvent le fruit de préjugés institutionnels dissimulés dans des politiques, pratiques et processus qui favorisent ou désavantagent les personnes en fonction de la race. La perpétuation de certains modes de fonctionnement peut également être en cause, si personne ne s'interroge sur la façon dont ils peuvent affecter certains groupes en particulier (ONTARIO, 2017). 
Le racisme d'État (ou institutionnel ou systémique) ne doit donc pas être confondu avec un État raciste. Un État raciste a une législation ouvertement raciste. Le racisme d'État concerne le fonctionnement institutionnel de l'État qui provoque des discriminations indirectes. Par exemple, l'État en France a été condamné pour des contrôles au faciès abusifs, par la police, de personnes racisées.

Là encore, il nous a paru tout à fait intéressant de faire connaître les travaux en pédagogie d'auteurs qui s'inscrivent dans la continuité de Paulo Freire relativement à cette thématique: concernant la pédagogie anti-raciste (CARR; THESEE, 2019) ou encore la pédagogie décoloniale... Cela d'autant plus que la réception du décolonial en France s'est faite de manière très biaisée via certains milieux militants. Les concepts issus de la théorie décoloniale latinoaméricaine ou de l'oeuvre de Boaventura de Sousa Santos sont utilisés, mais sans que les références aux auteurs qui les ont forgés soit explicitée. On peut parler d'un extractivisme cognitif qui réitère la domination eurocentrique. C'est pourquoi, il nous a paru intéressant de faire référence par exemples au travaux de Catherine Walsh en Équateur qui avait travaillé avec Paulo Freire et qui s'est inspirée entre autres de lui pour fonder la pédagogie interculturelle critique ou pédagogie décoloniale.

\subsection{Obligation de Neutralité et Obligation de Réserve.}

Si la situation en France et au Brésil est très différentes, on peut néanmoins remarquer d'étonnants points communs. Si le gouvernement au Brésil est classé comme étant d'extrême droite, celui en France, pourrait être plutôt qualifié par les philosophes ou les politologues de « libéralisme autoritaire »(CHAMAYOU, 2018). Pourtant, en dépit de cette différence on constate quelques tendances qui illustrent peut être moins la parenté directe entre les deux gouvernements qu'un glissement international vers des formes de régimes plus autoritaires. Ainsi, «l'Ecole sans parti » au Brésil veut faire passer une loi qui impose la neutralité aux enseignants, en France, le Ministre de l'Education nationale a fait voter une loi à l'hiver 2019, intitulé Ecole de la confiance, dans laquelle l'article premier met en avant un devoir d'exemplarité des enseignants visant à éviter tout dénigrement des politiques publiques de l'institution y compris en dehors de heures de service des enseignants. Il faut dire que l'obligation de neutralité imposée aux fonctionnaires existe déjà en France dans la loi depuis 2016. Au Brésil, le Ministre de l'Education nationale a incité les directeurs d'Ecole à faire chanter par les élèves l'hymne national et à filmer les élèves. En France, les députés ont introduit dans la loi dite de l'Ecole de la Confiance, un amendement proposant que dans toutes 
les salles de classes soit affiché le drapeau français, le drapeau européen et l'hymne français de la Marseillaise.

La manière dont nous avons interprété les attaques répétées de Jair Bolsonaro, contre l'oeuvre de Paulo Freire, est la suivante. Ce qui pose en réalité problème à Jair Bolsonaro, c'est une éducation qui se donne pour objectif de lutter contre les discriminations et d'éduquer aux droits humains. Ses propos racistes, sexistes et homophobes à différentes reprises durant la campagne électorale montrent qu'il ne partage pas un projet humaniste. Vouloir imposer la neutralité aux enseignants dans un tel contexte au Brésil, c'est rendre possible de considérer qu'un enseignant ou une enseignante qui défend la lutte contre les discriminations et les droits humains dans le cadre de ses missions porterait atteinte à son obligation de neutralité.

Cela nous a conduit à nous interroger sur la portée de l'obligation de neutralité en France des enseignants et de leur obligation de réserve. Comment se situe-t-elle par rapport au droit de la non-discrimination? En effet, un rapport du Conseil de l'Europe souligne que les personnalités politiques en France, y compris des élus, n'hésitent pas à tenir des propos discriminatoires ou à reprendre des stéréotypes négatifs à l'égard de groupes sociaux minoritaires: «le discours politique est sujet à une exploitation récurrente de déclarations stigmatisant les groupes vulnérables, et qui contribue à banaliser les attitudes racistes et intolérantes au sein de la population » (ECRI, 2016, p. 9).

En vertu de la hiérarchie des normes, qui fait que le droit européen et international, lorsqu'il sont transposés en droits internes, sont supérieurs au droit national, nous avons défendu la thèse que l'obligation de neutralité et le devoir de réserve d'un enseignant ne peuvent lui être appliqué du moment qu'il prend des positions visant à défendre le droit de la nondiscrimination et les droits humains.

Cela nous a conduit à défendre dans l'espace français, que la pédagogie de Paulo Freire ne rentrait pas en contradiction avec l'obligation de neutralité des enseignants. En effet, dans les textes de l'Education nationale que les enseignants doivent appliquer, il y a l'article L.1111 du Code de l'Education qui dit que les enseignants doivent lutter contre les inégalités sociales et le référentiel de compétence des enseignants, en application du droit européen, qui affirme que les enseignants doivent se mobiliser contre toutes les discriminations.

Cela est une rupture avec la lecture dominante, y compris dans les milieux progressistes, de considérer qu'il est incompatible avec la formation de l'esprit critique des élèves de considérer que l'enseignant peut énoncer son option comme l'affirme Paulo Freire (FREIRE, 2006). En effet en France, la formation à l'esprit critique a tendance à être conçue de manière technicienne. Il s'agit de donner aux élèves les outils, considérés comme neutres, pour penser 
par eux-mêmes. Mais l'enseignant ne doit pas défendre de positions car il pourrait influencer le choix des élèves.

L'intérêt de la position de Paulo Freire est de situer sa solution au niveau de la posture éthique de l'enseignant. Celui-ci affirme ses options - lutte contre les inégalités sociales et les discriminations -, mais son éthique de la liberté de l'apprenant consiste à développer les conditions d'un dialogue dans lesquels les élèves peuvent ne pas être d'accord avec cette option:

\begin{abstract}
Quand je travaille avec des enfants, je dois être attentif au passage ou au cheminement difficile de l'hétéronomie vers l'autonomie. Je dois être attentif à la responsabilité de ma présence qui peut tout aussi bien aider que perturber la quête inquiète des apprenants. (...) Je ne peux lui nier ou lui cacher ma posture, mais je ne peux, non plus, méconnaître son droit de la rejeter (FREIRE, 2006, p. 85).
\end{abstract}

\title{
Conclusion
}

Réintroduire la pensée de Paulo Freire en France actuellement nous a paru important pour répondre aux défis du néolibéralisme et du néoconservatisme. Nous avons en nous appuyant sur la pensée de Paulo Freire et de ses continuateurs essayé de mettre en avant plusieurs points.

Il est nécessaire comme le fait Paulo Freire d'affirmer des finalités d'émancipation sociale claire pour résister au néolibéralisme. Des pratiques pédagogiques actives ne suffisent pas à distinguer une pédagogie néolibérale et d'une pédagogie d'émancipation sociale.

La résistance à la domination de la raison technicienne en pédagogie passe par la réaffirmation claire des finalités d'émancipation sociale de la pédagogie. Mais en outre, cela suppose un refus d'un rapport instrumental aux moyens pédagogiques. Cela consiste à considérer que les pratiques pédagogiques ne relèvent pas de l'agir instrumental, mais d'un agir éthique. La finalité humanisante de l'éducation ne peut supposer le recours à des pratiques déshumanisantes.

Contre le néoconservatisme qui affirme une apparente neutralité de l'éducation pour en réalité la mettre au service du néolibéralisme, Paulo Freire affirme le caractère engagé de toute pédagogie dans la lutte contre les discriminations et les inégalités sociales. C'est la posture éthique qui permet de faire que l'affirmation de ses options par l'enseignant ne se réduise pas à un embrigadement, mais permette le développement de l'esprit critique via une éthique de la discussion (comme l'a également mis en avant Habermas). 
Enfin, en nous appuyant sur les continuateurs de Paulo Freire, nous avons pu davantage ouvrir la réflexion pédagogique en France à des domaines tels que la pédagogie féministe, critique de la norme, anti-raciste ou encore décoloniale.

\section{Références}

ADORNO Theodor, W. Modèles critiques. Paris: Payot, 1984.

CHAMAYOU, Grégoire. La société ingouvernable : une généalogie du libéralisme autoritaire. Paris. Editions : La Fabrique. 2018.

CHAMBAT, Grégory. L'école des réac-publicains. Paris : Libertalia, 2016.

CHEVARIN, Alain. ; CHAMBAT Grégory. De Montessori aux neurosciences. N'autre école. Hors Série n¹., 2019.

COLLET, Isabelle.«Dépasser les « éducations à » vers une pédagogie de l'égalité en formation des enseignants ». Pereira I. (dir.) Anthologie internationale de Pédagogie critique. Vulaines Sur Seine, 2019.

DE COCK Laurence. ; PEREIRA, Irène (dir.). Pédagogies critiques. Marseille: Agone, 2019.

DEVIEILHE Elise. La pédagogie critique des normes : apports et exemples suédois. Vulaines Sur Seine : Editions du Croquant. 2019.

FREIRE, Paulo. Pédagogie de l'autonomie. Toulouse: Eres, 2003.

FREIRE, Paulo. Pedagogia da indignação: cartas pedagógicas e outros escritos. São Paulo: Editora UNESP, 2000.

HIRTT, Nico. L'approche par compétences: une mystification pédagogique. L'école démocratique, 39, 1-34, 2009.

GIROUX, Henry. (2018). Pedagogy and the politics of hope: theory, culture, and schooling: a critical reader. New York: Routledge, 2018.

LAVAL, Christian. L'école n'est pas une entreprise: le néo-libéralisme à l'assaut de l'enseignement public. Paris: La Découverte, 2003.

MEDEF (2012). «Pour une école exigeante, personnalisée et créative ».URL : http://www.medeflyonrhone.fr/2012/10/16/contribution-du-medef-a-la-concertation-sur-larefondation-de-lecole/

MEIRIEU, Philippe. La Riposte. Paris : Autrement, 2018. 
ONTARIO. Plan stratégique triennal contre le racisme. URL : https://www.ontario.ca/fr/page/une-meilleure-facon-davancer-plan-strategique-triennal-delontario-contre-le-racisme, consulté à mars 2017.

PEREIRA, Irène. Paulo Freire - Pédagogue des opprimé-es. Paris: Libertalia, 2017.

PEREIRA, Irène. Bréviaire des enseignant-e-s-: Science, éthique et pratique professionnelle. Vulaines Sur Seine: Éditions du Croquant, 2018.

PEREIRA, Irène. Philosophie critique en éducation. Limoges: Lambert-Lucas, 2018.

PEREIRA I. (dir.). Anthologie internationale de pédagogie critique. Vulaines Sur Seine: Editions du Croquant, 2019.

SAINT-VINCENT Lise-Anne (dir.). Le développement de l'agir éthique chez les professionnels de l'éducation. Montréal : Presses Universitaires du Quebec.

SOLAR, Claudie. "Dentelle de pédagogie féministe». In Pereira I. (dir.) Anthologie internationale de pédagogie critique. Vulaines Sur Seine : Editions du Croquant, 2019.

THESEE, Gina ; CARR, Paul R. La (re) lecture des mots, du monde et des maux des jeunes noirs: apports de la pédagogie critique à la recherche en éducation en contextes de racialisation. In Pereira I. (dir.). Anthologie internationale de pédagogie critique. Vulaines Sur Seine: Editions du Croquant, 2019.

TOLINI, Gauthier. Freinet: de Marx à Bill Gates. Site des cahiers de pédagogie radicale. URL : https://pedaradicale.hypotheses.org/1842, consulté à mars 2018. 